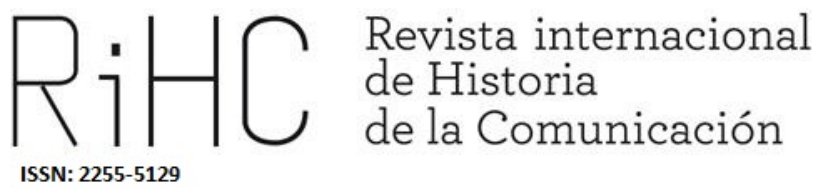

\title{
ENTRE LA INFORMACIÓN Y LA CREACIÓN DE IDENTIDAD: ORIGEN Y DESARROLLO DE LA PRENSA DE LA EMIGRACIÓN VASCA EN AMÉRICA, 1877-1936
}

Between Information and Creation of Identity: Origin and Development of the Press of Basque Emigration in the Americas, 1877-1936

DOI: http://dx.doi.org/10.12795/RiHC.2019.i12.02

Recibido: $12 / 03 / 2019$

Aceptado: 05/04/2019

Publicado: $15 / 06 / 2019$

Óscar Álvarez Gila

Universidad del País Vasco, España

oscar.alvarez@ehu.eus

ORCID (iD) 0000-0002-8829-5207 
Resumen: En el artículo se hace una presentación general sobre el desarrollo de la prensa de la diáspora vasca desde su nacimiento en la década de 1870 hasta la Guerra Civil española. En primer lugar, se analiza el estado de la cuestión sobre los estudios de este tipo particular de prensa, y muy especialmente el efecto positivo que tuvo en la investigación la digitalización y puesta a disposición de los investigadores de la hemeroteca digital de la diáspora vasca promovida por el Gobierno Vasco hace una década. Seguidamente, se hace un estudio de las cabeceras que se crearon, definiendo una caracterización en base a los promotores y sus líneas ideológicas subyacentes. Finalmente se presentan algunas precisiones preliminares sobre el impacto que el desarrollo de esta prensa tuvo en la definición (así como las siguientes modulaciones a lo largo de las décadas) de la idea de identidad vasca, expresada de forma dialéctica desde formulaciones puramente regionalistas hasta otras próximas al nacionalismo vasco.

Palabras clave: Prensa vasca, emigración, regionalismo, nacionalismo, América

Abstract: This article offers a general presentation on the developmen of Basque diasporic press since its beginnings in the 1870s until the Spanish Civil War. First of all it is analyze the state of the art on the research on this particular topic, and more specially, the positive impact derived from the implementation of a digitized library of Basque diasporic press by the Basque Governmnent a decade ago. Next, a study on the periodicals is done, defining a characterization of their main features through who their promoters were and which their ideological lines were. Finally, some preliminary remarks are presented on the impact that the development of this press had in the emergence and evolution of Basque identity abroad, dialectically expressed from regionalistic to nationalistic views.

Keywords: Basque press, emigration, regionalism, nationalism, America

\section{Introducción y metodología: la "Hemeroteca de la Diáspora Vasca"}

Entre 2003 y 2007 se puso a disposición de los investigadores el resultado de más de cinco años de un trabajo de localización, catalogación y organización de un archivo de prensa o hemeroteca digital que, bajo el nombre de "Urazandi Digital", había sido promovido desde la Dirección de Relaciones con las Colectividades Vascas del Gobierno autonómico vasco (Irigoyen Artetxe y Patrón, 2003; Irigoyen Artetxe y Patrón, 2007). Editado inicialmente en dos colecciones de DVDs, su accesibilidad se vio ampliada poco tiempo más tarde al ser puesta la mayor parte de la colección accesible a través de internet ${ }^{1}$. Años más tarde el proyecto se vería completado con

1 Disponible en la dirección http://urazandi.euskaletxeak.net/default.html dentro del portal "Euskaletxeak" desarrollado por el propio Gobierno Vasco. En este enlace se puede consultar toda la 
una segunda fuente hemerográfica digitalizada, en cierto modo complementaria aunque diferente en su concepción y alcance, porque se centraba en la recopilación de referencias periodísticas sobre los vascos publicadas en la prensa generalista de los países en los que se asentó preferentemente la emigración (Álvarez, Vitullo y Echezarreta, 2009) ${ }^{2}$.

Se abría así campo de nuevas posibilidades para toda persona interesada en realizar investigaciones en torno a lo que en las últimas décadas ha venido de denominarse como la "diáspora vasca", es decir, el campo temático que abarca los estudios sobre la emigración, presencia y acción de personas y colectividades de origen vasco establecidas en diversos países, muy especiamente en el continente americano, en su mayor parte como consecuencia de las emigraciones masivas que tuvieron lugar a lo largo de los siglos XIX y XX, entre las que se ha desarrollado un proceso mantenimiento, reelaboración y en algunos casos recuperación de la identidad vasca de sus ancestros (Totoricaguena, 2005: 26-30). Con una intención de recuperar la totalidad de las publicaciones periódicas editadas en el exterior por los vascos ( $\mathrm{y} / \mathrm{o}$ sobre temática vasca ${ }^{3}$ ) en los siglos XIX y XX, la digitalización abarca un total de 133 cabeceras diferentes, datadas entre 1877 (la más antigua) y 2006 (la más reciente) ${ }^{4}$, procedentes de 13 países diferentes (Tabla $n$ o 1).

\section{Tabla no 1: Número total de publicaciones periódicas digitalizadas en la Hemeroteca de la Diáspora}

\begin{tabular}{|l|l|l|}
\hline País & Nocabeceras & Fechas límite \\
\hline Uruguay & 6 & $1877-1985$ \\
\hline Argentina & 38 & $1878-2003$ \\
\hline Estados Unidos & 14 & $1885-2006$ \\
\hline
\end{tabular}

colección de revistas, excepto La Baskonia de Buenos Aires (1893-1943), que por ahora solo puede ser consultada en el DVD originalmente editado.

2 Disponible en la dirección http://vpa.euskaletxeak.net dentro del portal "Euskaletxeak" desarrollado por el propio Gobierno Vasco.

3 Quedó fuera de esta colección la colección de Oficina de Prensa de Euzkadi / Euzko Izpar-Banatzea, más conocido por sus siglas en castellano (OPE), que fue el órgano oficial para los comunicados de prensa del Gobierno Vasco en el exilio, editándose entre 1947 y 1977 . Se halla igualmente disponible la digitalización completa en el mismo portal web, en la dirección http://ope.euskaletxeak.net

4 La digitalización no ha podido abarcar todos los números realmente publicados, ni todas las cabeceras que en un momento $u$ otro vieron la luz, por haberse perdido y no saberse de su existencia, a la fecha de elaboración del proyecto, en ninguna de las grandes bibliotecas, hemerotecas y colecciones particulares de referencia en las que se realizó, tanto en Europa como en América. Con posterioridad se han encontrado ejemplares no incluidos en la base de datos, que el Gobierno Vasco tiene intención de digitalizar e incorporar a la hemeroteca digital en un plazo breve. Este trabajo se realiza sobre las obras incluidas en la hemeroteca a fecha enero de 2019. 


\begin{tabular}{|l|l|l|}
\hline Cuba & 7 & $1886-1972$ \\
\hline Chile & 4 & $1907-1960$ \\
\hline México & 23 & $1907-1975$ \\
\hline Reino Unido & 2 & $1937-1938$ \\
\hline Francia & 4 & $1939-1972$ \\
\hline Panamá & 1 & 1943 \\
\hline Venezuela & 28 & $1946-1981$ \\
\hline Guatemala & 1 & $1950-1959$ \\
\hline Argelia & 1 & 1958 \\
\hline Colombia & 2 & $1962-1977$ \\
\hline
\end{tabular}

Fuente: Irigoyen Artetxe y Patrón, 2003 y 2007.

\section{Estado de la cuestión}

Si bien existieron con anterioridad trabajos que tomaron como aspecto parcial de estudio la prensa de la diáspora vasca, hubo que esperar sin embargo en gran medida a la publicación de esta hemeroteca digital para que surgieran estudios específicos sobre la historia de la prensa de la diáspora vasca. Por lo general, la mención a revistas, almanaques u otras publicaciones periódicas solían verse limitadas a su uso limitado como fuente para la elaboración de investigaciones históricas de diversa temática y alcance, pero que en ningún caso tenían a la propia prensa como su objeto central de análisis ${ }^{5}$. Solo cabe señalar como excepción, en la década de 1990, los trabajos pioneros de Díaz-Noci (1994; 1995), si bien en su caso su aproximación al análisis de la prensa de la diáspora vasca vino derivada de su especialización en la historia de la prensa vasca, y no en en la historia de la diáspora. De este modo en ambos trabajos, en cierto modo relacionados en su génesis y concepción, Díaz-Noci pretendía hacer un estudio sobre los antecedentes históricos de la prensa escrita en lengua vasca, a fin de mostrar que, si bien en el panorama presente y pasado de los medios de comunicación de masas su presencia ha sido y es minoritaria -en relación con las mucho más abundantes empresas periodísticas, y luego radiofónicas y

5 Por ejemplo, en la obra que Azcona Pastor dedicó a hacer un estudio general sobre la emigración vasca en Argentina y Uruguay, la prensa vasco-uruguaya y vasco-argentina apenas merece sendas menciones de apenas dos líneas, dentro de un apartado general dedicado a la "conservación de los rasgos étnicos" (Azcona Pastor, 1992: 247 y 327), si bien este autor reconoce a lo largo de la misma obra la importancia de la prensa como fuente al usarla profusamente en el estudio de la opinión pública vasca frente a la emigración. 
televisivas en castellano o francés-, no por ello no habían dejado de existir intentos, más o menos exitosos, de publicaciones periódicas dirigidas al público vascoparlante. Fue así como se encontró con la pista de dos cabeceras periodísticas, íntegramente publicadas en euskara, que vieron a la luz en Los Ángeles, California, en el último cuarto del siglo XIX: Escualdun Gazeta (1885-1886) y California-ko Eskual Herria (18931897), dirigidos respectivamente por los vasco-franceses Martin V. Biscailuz y JeanPierre Goytino; sobre los que realizó un estudio descriptivo pormenorizado de su génesis, contenido temático, evolución y otros aspectos propios de su carácter de prensa específicamente dirigida a un colectivo inmigrante, como eran los vascos (DíazNoci, 2001). Díaz-Noci, y posteriormente otros autores como Arbelbide (2001; 2003; 2006), quien se centró específicamente en la biografía y la labor de Goytino ${ }^{6}$, pusieron principalmente de relieve la gran novedad que suponía, en el panorama de los emprendimientos periodísticos en el País Vasco, la aparición en un ámbito geográfico tan lejano y específico de dos cabeceras que eran, por ese orden, la segunda y tercera publicación periódica escrita únicamente en euskara en toda la historia de la comunicación periodística vasca.

No obstante, como hemos señalado, no fue hasta que la digitalización de la prensa de la diáspora vasca viera a la luz que comenzaría realmente una línea de estudios, todavía incipientes, aunque no por ello menores, sobre el objeto, papel, evolución y contenido de esta prensa como ámbito particular de nuevas investigaciones. Entre otros, pueden citarse trabajos como los de Irigoyen Artetxe (2010), Ramos Martínez (2010; 2011; 2017) o Mehats (2012), que han trabajado sobre ejemplos concretos del periodismo vasco en Uruguay, Cuba o Argentina, respectivamente. También se incluyen en este grupo los estudios sobre la más longeva de las cabeceras de la prensa vasco-americana: La Baskonia de Buenos Aires, que salió a la luz puntualmente con una periodicidad de tres números al mes entre 1893 y 1943 (Bergareche, 2009); y más en concretamente sobre quienes actuaron como responsables de la empresa y firmaron obras periodísticas o artísticas de las que aparecieron en sus páginas, en especial el escritor Francisco Grandmontagne (Ares, 2004); el editor José Rufo de Uriarte (De Dios Altuna y Álvarez Gila, 2010), la pintora Andrea Moch (De Dios Altuna, 2011) o el fotógrafo Indalecio Ojanguren (De Dios Altuna, 2012). Igualmente, la fácil disponiblidad de las fuentes en su versión digitalizada está igualmente permitiendo la elaboración de trabajos académicos universitarios, vinculados a proyectos de fin de grado o de máster, de alumnos interesados de la Historia (Perea Beobide, 2017) o los estudios de Comunicación (Zelaia, 2018).

6 Que el interés primario del trabajo de Arbelbide viniera dado por el carácter de su biografiado como promotor de la lengua y cultura vasca viene dado por la colección en la que le fue encargada la elaboración de la biografía (2003) y donde fue posteriormente publicada: "Bidegileak" (es decir, los "Abridores de camino"), que está promovida y editada por el departamento de promoción del euskera del Gobierno Vasco. 
Este último trabajo tiene, además, la virtud de ser al mismo tiempo una de las primeras reflexiones omnicomprensivas del fenómeno de la prensa vasca de la diáspora ( $y$, en extensión y profundidad, claramente superior a la de Torregrosa Carmona, 2010), así como un proyecto detalladamente elaborado para una futura tesis doctoral sobre la materia. Sus dos hipótesis principales son, por un lado, que el crecimiento de la prensa significaría "que debía existir una masa crítica en el seno de las comunidades vascas" que permitiera su aparición y continuidad; y por otro lado, que existe una clara diferenciación en las características que presenta el panorama de la prensa de la diáspora antes y después de la Guerra Civil española, que llevó a "una creciente politización de los contenidos" y los emprendimientos periodísticos por el impacto que tuvo la recepción del exilio (Zelaia, 2018: 6). En este artículo discutiremos alrededor de ambas hipótesis.

\section{Evolución: los hitos de un crecimiento}

Como ya hemos indicado anteriormente, la aparición de una prensa de la diáspora vasca es una consecuencia de la propia existencia de dicha diáspora, es decir, de la formación de grupos o colectividades organizadas y dotadas de una particular organización, estructura formal e identidad, que nucleaban a inmigrantes procedentes del territorio vasco radicados en aquellos países que, por diversas razones, habían atraído los mayores contingentes. La prensa se constituía así no solo como medio de comunicación interna de dichas colectividades, sino también como uno de los espacios simbólicos más importantes para estructurar unos colectivos que, por lo general, no presentaron pautas de radicación en ghettos o barrios étnicos estrictos. Como ya hacía notar en 1910 Pierre Lhande, "dans la plupatrt des régions où ils se rencontrent une poignée, les émigrés euskariens fondent leur petit journal basque; ilts bâtissent un fronton pour le jeu de pelote" (Lhande, 1910: 161-162).

Es preciso reconocer, de todos modos, que la prensa específicamente vasca no fue el único nexo formal usado para la comunicación en el seno de esta colectividad. Debido a la diversidad de sus orígenes y a la existencia en su seno de ámbitos de pertenencia identitaria divergentes y complementarios, muchos vascos utilizaron como sistemas de comunicación de la colectividad, antes y después de la aparición de la prensa vasca, medios de comunicación identificados como órganos de expresión de las colectividades española y francesa. De hecho, ya desde los momentos iniciales de la nueva emigración masiva hacia América a mediados del siglo XIX se constató la presencia de artículos, comunicados, llamados y avisos dirigidos específicamente a los vascos en publicaciones de la prensa étnica de ambas colectividades en países como Argentina o Uruguay. Por ejemplo, los primeros artículos periodísticos redactado 
íntegramente en lengua vasca fueron publicados el año 1843 en Le Patriote Français de Montevideo a lo largo de 1843 (Mehats, 2002. Irigoyen Artetxe, 2010: 207-208). Esta presencia, de hecho, se aprecia incluso en diarios y revistas que no entran en la categoría de la prensa étnica de los inmigrantes. Así, por ejemplo, Irigoyen Artetxe (2010: 215-217) menciona el caso del diario El Pueblo, publicado en la localidad uruguaya de Santa Isabel de Paso de los Toros, y que en sus palabras se convertiría, "sin duda alguna, [en] el órgano de prensa del colectivo vasco de aquella villa". Este hecho se hizo visible cuando "a partir de 1912 la sociedad Euskal Erria comenzó a insertar en su periódico social una serie de artículos firmados (..) con el nombre del autor, Valentín del Escobal, o con el seudónimo de 'Un vizcaíno'", dado que del Escobal era asimismo el propietario y director del mencionado diario El Pueblo, en cuyas páginas tuvieron cumplida presencia las noticias dirigidas a la comunidad vasca local, hasta su fallecimiento en 1920

En todo caso, tanto la diversidad geográfica de la prensa diaspórica vasca (según se refleja en la tabla no 1), como su cronología de aparición y la evolución del número de cabeceras periodísticas surgidas en cada país constituyen un buen reflejo de la imagen general que tenemos sobre la propia evolución de la emigración vasca en los siglos $X I X$ y XX, uniendo bajo este paraguas no solo las que se han considerado como migraciones sensu stricto (es decir, motivadas por razones de tipo principalmente económico), sino también los exilios causados por persecuciones políticas, especialmente los que se produjeron tras los diversos episodios bélicos que afectaron los territorios vascos a lo largo de este periodo. Entre ellas destaca principalmente, por su elevado impacto en la producción periodística, el exilio de los vascos leales al primer Gobierno Vasco y a la legalidad republicana tras su derrota en la Guerra Civil española de 1936-1939.

Tabla no 2: Cabeceras que aparecieron antes de 1936 (inicio de la Guerra Civil)

\begin{tabular}{|c|c|c|c|}
\hline País & $\begin{array}{c}\text { Cabeceras } \\
\text { hasta } \mathbf{1 9 3 6}\end{array}$ & $\begin{array}{c}\text { Total } \\
\text { cabeceras }\end{array}$ & \% sobre el total \\
\hline Argentina & 14 & 38 & 36,84 \\
\hline Uruguay & 5 & 6 & 83,34 \\
\hline Cuba & 4 & 7 & 57,14 \\
\hline México & 4 & 23 & 17,39 \\
\hline Estados Unidos & 3 & 14 & 21,43 \\
\hline Chile & 1 & 4 & 25 \\
\hline Resto países & 0 & 102 & 0 \\
\hline
\end{tabular}

Fuente: Irigoyen Artetxe y Patrón, 2003 y 2007. 
De hecho, si establecemos una división entre las publicaciones editadas antes y después del año 1936 (Tabla no 2), comprobaremos que las que aparecieron con anterioridad al inicio de la Guerra Civil (31) apenas representan un 23,30\% del total de cabeceras registradas. También se aprecia, igualmente, una menor diversidad geográfica de procedencia de los diferentes diarios y revistas antes de 1936. Los diferentes porcentajes que representan estas cabeceras frente al total, analizados país a país, nos muestran dos tipos de modelos. Todos ellos son países que contaban una larga tradición inmigratoria vasca desde momentos tempranos en el siglo XIX, pero que habían sufrido una ralentización de las llegadas de nuevos contingentes tras la crisis de 1929. No obstante, por un lado se encontraba el caso de Uruguay, y en menor medida de Cuba, en los que las cabeceras previas a la guerra representan más de la mitad del total, lo que venía a reflejar que al finalizar la Guerra Civil española no se convierten en territorios que recibian contingentes amplios de exiliados. Argentina, México o Chile representan, por su parte, el ejemplo contrario, ya que fueron países en los que se concentró una fuerte presencia del exilio ${ }^{7}$.

Por supuesto, estas cifras habrían de ser matizadas si entramos a analizar la tipología de las publicaciones periódicas recogidas en esta lista. No podemos inferir, como hace Torregrosa Carmona (2010), que todas estas publicaciones entren en la categoría de lo que se entiende habitualmente como prensa. Frente a la imagen concreta que evoca el uso popular de este término, realmente resulta difícil realizar una definición precisa y, sobre todo, marcar unos límites demasiado estrictos que señalen la frontera entre lo que es y lo que no es considerable como prensa. Si usamos la definición establecida por Alonso (2004) cuando habla de la prensa como un conjunto de "construcciones escritas" de gran diversidad de formas (incluyendo panfletos, revistas y diarios, entre otros medios) y con un objetivo común de difusión de ideas y opiniones vinculadas, más o menos directamente, a la actualidad, rápidamente nos percatamos de que sería muy cuestionable identificar en esta categoría varias de las publicaciones recogidas en el listado. Así, por ejemplo, la publicación periódica vasca más longeva de las incluidas en la "hemeroteca de la diáspora", iniciada en 1890 y aún activa en 1972, titulada Memoria de la Asociación Vasco-Navarra de Beneficencia, realmente no es otra cosa que la versión impresa del balance económico, de socios y de actividades presentado en la asamblea anual de dicha asociación con sede en La Habana (Cuba); es decir, un producto impreso que nadie osaría identificar como órgano de prensa escrita según las definiciones más aceptadas.

7 Venezuela sería, de hecho, el caso extremo de este grupo, ya que carecía de una inmigracion económica vasca apreciable antes de la guerra, mientras que aceptó un grupo numeroso y compacto de exiliados nacionalistas a su finalización: las numerosas cabeceras publicadas en Venezuela son todas, de hecho, publicaciones vinculadas al exilio. Por su parte, Estados Unidos es la única excepción, ya que no recibió exiliados, por lo que el elevado porcentaje de publicaciones vascas que aparecieron en ese país con posterioridad de 1936 lo que vendrían a reflejar es, principalmente, el mantenimiento de la corriente migratoria económica, que se mantuvo hasta fecha tan tardía como la década de 1970. 


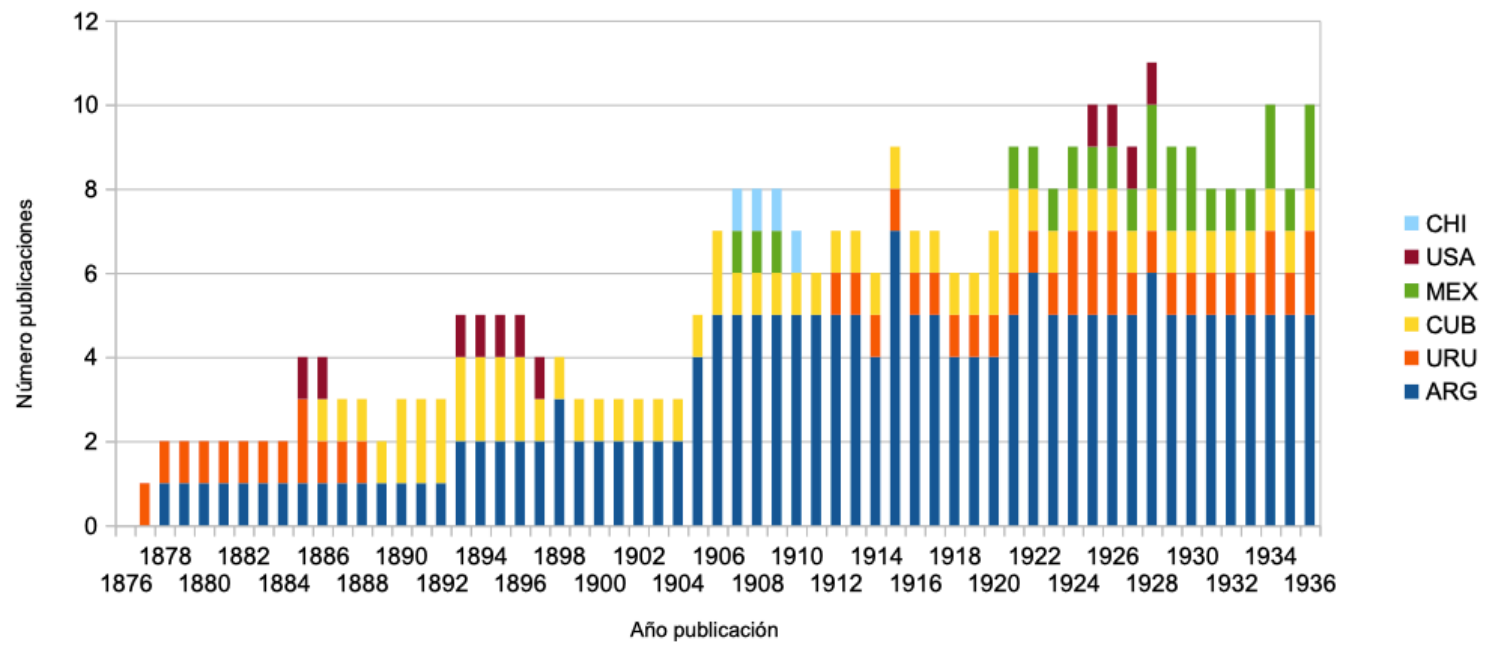

Gráfico no 1: Evolución de las cabeceras activas año por año en las publicaciones periódicas de la diáspora vasca, desglosadas por países (1877-1936).

También es importante matizar las cifras brutas del número de publicaciones periódicas aparecidas en la diáspora, porque dichos datos sitúan en pie de igualdad empresas periodísticas que duraron décadas (como La Baskonia de Argentina en la primera etapa, o las diversas ediciones americanas de Euzko Deya, el principal órgano de comunicación del exilio nacionalista vasco, en la segunda etapa), con otras que apenas pasaron de sacar a la luz unos pocos ejemplares antes de desaparecer, o se mantuvieron con una gran irregularidad. Analizando de este modo la permanencia de cada una de las publicaciones, hemos obtenido la evolución que se aprecia en el Gráfico no 1, que recoge de modo acumulado aunque desglosado por países la evolución entre 1877 y 1936.

Un elemento que destaca en esta evolución es la progresión al alza en el número total de las publicaciones periódicas. De hecho, aunque como hemos señalado son muy pocas entre ellas las que presentan un contunidad que vaya más allá de la década, sin embargo el panorama general nos muestra una notable vitalidad: a las que desaparecen con el paso del tiempo, le suceden prontamente otras iniciativas editoriales que vienen pronto a cubrir un nicho de mercado que, según se muestra, parecía ser lo suficientemente maduro como para sostener todas ellas. En cierto modo, esta evolución presenta un panorama muy similar al que ofrecen otras iniciativas formales de la colectividad vasca, y más en concreto, su proceso de institucionalización mediante la formación de asociaciones étnicas (los conocidos habitualmente como "centros vascos" o más recientemente, en la historiografía, con su denominación en euskera, euskal etxeak). A este respecto, se puede establecer con la prensa una periodización muy similar a la que cabe establecer con las euskal etxeak, es decir: un periodo inicial de creación y florecimiento de una primera generación, tanto de asociaciones como de cabeceras periódicas, en las décadas de 1870 y 1880; 
un momento de aparente decadencia a lo largo de la década de 1890 que llevaría a la desaparición de algunas de ellas; un segundo florecimiento asociativo y editorial a partir de mediados de la primera década del siglo XX, y un crecimiento sostenido hasta los inicios de la década de 1930 -en la que se dejarían ver los efectos de la crisis de 1929 y sus implicaciones negativas en los procesos migratorios ultramarinos-.

\section{Las empresas periodísticas: promotores, público, objetivos}

De hecho, este paralelismo nos da pie a analizar otra de las características de las iniciativas editoriales que dieron lugar a todas estas publicaciones que conforman la hemeroteca de la diáspora vasca: las personas, grupos e instituciones promotoras que se hallaban detrás de su puesta en marcha. Las iniciativas periodísticas vascoamericanas que surgieron en el intervalo analizado pueden de este modo clasificarse en dos grandes grupos:

a) Por un lado, y en primer lugar, se encuentran lo que podríamos denominar el periodismo institucional, es decir las revistas, boletines y órganos de expresión de los centros vascos, que los crean con el objetivo principal de que sirvieran como su medio habitual para la comunicación con la masa societaria (Zelaia, 2018: 29). La edición de boletines, revistas periódicas y otros medios de comunicación escritos para la información de socios y simpatizantes como uno de los ámbitos más comunes de actuación de los centros vascos, ya fue un hecho puesto de relieve por Márquez Ortiz (1996) y Cava Mesa (1996).

De hecho, existe una notable coincidencia entre la fecha de aparición de las primeras instituciones asociativas de la inmigración vasca y el momento en que dichas asociaciones deciden dar a la luz sus propias revistas: los dos primeros centros vascos de la región del Río de la Plata, ambos denominados Laurak Bat, en Montevideo y Buenos Aires, optan por poner en marcha sendos boletines durante su primer año de existencia, en 1877 y 1878, respectivamente (Irigoyen Artetxe, 2000: 80; Ezkerro, 2003: 31). Ambas revistas recibieron como título el mismo nombre de la asociación, un rasgo que se repetirá posteriormente cuando otros centros similares creen sus propias revistas societarias: por ejemplo, el Zazpirak Bat de Rosario, cuya revista de igual título nace en 1922 (Álvarez Gila, 2000), o el Euskal Erria de Montevideo (en 1912).

En el caso de Uruguay, por ejemplo, cuatro de las cinco cabeceras registradas por Irigoyen Artetxe (2010) entre 1876 y 1939 corresponden realmente a este tipo de 
periodismo interno vinculado a instituciones del asociacionismo vasco. Más aún, este mismo autor cita además otras dos posibles cabeceras, de las que no se habrían conservado ejemplares, pero que por las descripciones que tenemos se corresponderían igualmente a esta modalidad. Así, según un testimonio tardío publicado "en Euzko Deya de Buenos Aires" con relación a la sociedad vasca Euskaldunak Bar de San José de Mayo, fundada en 1877, se "decía que esta euskal etxea distribuía una pequeña hoja redactada exclusivamente en euskara" (Irigoyen Artetxe, 2010: 212). Careciendo de otra referencia no podemos calibrar el alcance y contenido de esta hoja, si bien se enmarca perfectamente en esta práctica que vinculaba la creación de un centro vasco con la edición de un órgano propio de difusión. Del mismo modo cuando en 1896 se refunda en Montevideo la sociedad Laurak Bat-habiendo desaparecido su precedente por bancarrota, pocos años antes, la renovada entidad también reanudó la edición de un boletín, del que sin embargo no se ha conservado ningún ejemplar conocido (Irigoyen Artetxe, 2010: 213) ${ }^{8}$.

b) Por otro lado, en una segunda fase surgen lo que podemos denominar la prensa de iniciativa empresarial privada en el contexto de las publicaciones periódicas dirigidas a las colectividades de inmigrantes vascos. Son, en principio, menos numerosas que aquellas, y frente a lo que cabría aventurar, el hecho de que carecieran del respaldo institucional de una asociación no parece repercutir ni en su difusión, ni sobre todo en su permanencia temporal, ya en esta prensa empresarial nos encontramos tanto con ejemplos de iniciativas fugaces como con las cabeceras que consiguieron alcanzar una estabilidad de publicación durante muchas décadas. Entre las primeras, cabe citar el caso ya mencionado de las dos publicaciones aparecidas tempranamente en California, Escualdun Gazeta y California-ko Eskual Herria, que por ser producto de la iniciativa estrictamente personal de personas no dedicadas profesionalmente al periodismo solo permanecieron en activo mientras sus impulsores tuvieron tiempo y medios materiales para llevarlas adelante.

Entre las segundas destaca especialmente el caso del decenario La Baskonia de Buenos Aires, que destaca en este panorama como el emprendimiento más exitoso por su longevidad, y por la calidad editorial, sobre todo en el periodo en que lo dirigó José Rufo de Uriarte, uno de sus fundadores, y su director, redactor y dueño único y vitalicio hasta su fallecimiento en 1932 (De Dios Altuna y Alvarez Gila, 2010). Nacida en 1893 con el nombre de La Vasconia, que la identificó hasta 1903, su importancia en el panorama periodístico y en la conformación de la colectividad vasco-argentina fue reconodida por los coetáneos y avalada por investigadores recientes. "Il est imposible d'ércire sur les Basques en Argentine dans le période 1893-1938 san avoir lu cette publication qui mérite à son époque de grands éloges autant de la part des journalistes basques qu'argentins" (Mehats, 2005: 286). En gran medida, buena parte

8 No hemos incluido estas dos cabeceras en los cuadros y gráficos de este artículo. 
del éxito inicial de la revista viene dado por el tándem de redactores que le dieron nacimiento: junto con Uriarte, en el primer decenio de existencia fue codirector el reconocido periodista y escritor Francisco Granmontagne (Ares, 2004), cuya impronta se deja ver en el contenido y enfoque de los artículos que aparecen en la revista, como ya se anunciaba en el anuncio firmado por "La Dirección" en su primer número:

Nuestra revista, como ya anunciábamos en la circular-programa, apropósito de su fundacion, tendrá el doble carácter de euskaro-americana.

Entre las varias y poderosas razones que nos han inducido á imprimirle este carácter, cuéntase en primer término, (..) la ilustre pléyade de americanos de origen vascongado, que desde la fecha de la emancipacion hasta nuestros dias, han sido en todos los conceptos las figuras mas descollantes de sus respectivos paises (...).

La actual importancia comercial de nuestra laboriosa colonia, tanto aquí entre nosotros como en las Repúblicas limítrofes, es por fin, otra de las razones que han determinado nuestra decision en el sentido ya indicado.

A pesar de esto, nuestra publicacion se ocupará con preferencia de todo lo que se refiera á nuestras queridas provincias, dando á conocer sus hombres eminentes y reseñando al propio tiempo todos los sucesos que ocurran dignos de mencion ${ }^{9}$.

Esta declaración de intenciones mostraba un cierto caracter ecléctico en los objetivos de la revista, basculando entre una propuesta enfocada a la alta divulgación cultural -siguiendo modelos de revistas similares, como por ejemplo la longeva Ilustración Española y Americana de la que parece tomar, entre otros, el profuso uso de la imagen (grabados, y posteriormente fotografías) - y otra más vinculada a la información de actualidad. En cierto modo, esta dicotomía respondía a los intereses de sus coeditores, el literato Grandmontagne y el impresor Uriarte, este último el financiador de la empresa a través del negocio editorial de su propiedad, a lo que se sumaba "su vocacion por el periodismo" (De Dios Altuna, 2012b). En el primer decenio de existencia de La Vasconia, triunfaron los planteamientos de Grandmontagne. Como señala Cava Mesa (1996: 149), la revista pronto alcanzó gran difusión por toda Argentina, siendo sus lectores "generalmente vascos 'cultivados"'y sobre todo relacionados con los importantes centros vasco-argentinos que dispusieron regularmente de ejemplares en las respectivas bibliotecas o sedes sociales". El plantel de firmas literarias que aparecían en sus páginas contribuyó a acrecentar el prestigio de una revista más concebida como emprendimiento cultural que étnico:

9 "Nuestro propósito", La Vasconia, Buenos Aires, I:1, 10 de octubre de 1893, p. 4. 
En sus años de expansión, publicaron en ella prestigiosos escritores. Desde sus inicios la obra de Grandmontagne, fue señera. Durante los años que ejerció la codirección, publicó casi dos centenares de biografías firmadas con el seudónimo de Luis Jaizquibel o con las iniciales, L. J.; F. G. o sólo su apellido. A ello se agregan cuentos, capítulos de novelas y diversos ensayos Otros escritores de renombre fueron Unamuno, Pío Baroja, Carmelo de Echegaray, los argentinos Leopoldo Lugones, Lucio Mansilla, José Ingenieros, el nicaragüense Rubén Darío por mencionar sólo algunos. La revista difundió la gramática vasca del jesuita Manuel Larramendi, Arturo Campión y muchos otros. Recibía permanentes colaboraciones de Euskadi de novelistas, vascófilos lingüistas, poetas, ensayistas, y numerosos periodistas que en calidad de corresponsales o no, aportaron importante material de información contribuyendo a la difusión y promoción de la cultura vasca en la diáspora (De Dios Altuna, 2012: 647).

El cambio de nombre de la revista en 1903, unido al abandono por parte de Grandmontagne por diferencias con la línea editorial y política que estaba queriendo imprimirle Uriarte (Ares, 2004: 153-155), evolucionaría hacia un modelo de revista que, sin perder los artículos atemporales sobre historia, geografía, cultura o lengua vascas, introduciría mayor peso a las noticias de actualidad, tanto referidas al conjunto del Pais Vasco como a las diversas colectividades locales vasco-argentinas (Cava Mesa, 1996: 149-151).

La diferenciación entre ambos tipos de iniciativas periodísticas resulta clara en la formulación teórica, sobre todo en lo tocante al tipo de informaciones preferentes que cada uno de ellos tenía como objetivo difundir. Es decir, que mientras que la prensa institucional tendría como objeto principal la difusión de noticias internas de la vida y actividades de los centros vascos, con un público primario compuesto por asociados y simpatizantes, la prensa de empresarios privados optaría más por un modelo de difusión de artículos generalistas, vinculados a tres ámbitos principales: las noticias del País Vasco, la actualidad del país de acogida, y los artículos de corte literario y cultural. No obstante, si bien su aplicación práctica al estudio de las diversas revistas vasco-americanas que fueron surgiendo nos muestra que la realidad es mucho más compleja y que las tipologías no funcionan como categorías estrictas y totalmente diferenciadas. Las transformaciones que experimenta La Baskonia a partir de 1903, dando cabida en sus páginas a noticias de los centros vascos de Argentina, contando para ello con el paso del tiempo con una red de corresponsales oficiosos encargados de transmitir a la redacción las novedades que ocurrían en su seno, es una buena muestra de ello.

De hecho, entre las publicaciones que desde un principio se presentan como desvinculadas orgánicamente de cualquier centro vasco preexistente, las más 
abundantes son aquellas que podemos denominar como "de origen mixto", es decir, promovidas, dirigidas y financiadas en su mayor parte por empresarios externos, pero que de algún modo nacen con una cierta vinculación, difusa o más institucionalizada, con el asociacionismo. En Cuba se halla el primer ejemplo paradigmático de esta práctica: la revista Laurak Bat de La Habana (1886-1896). Como recogen los trabajos de Ramos Martínez (2010; 2011), esta revista surgió de la conjunción de intereses entre su director, Faustino Díez Gaviño, y el financiador de la empresa, su tío y enriquecido indiano Manuel Calvo y Aguirre, quien aportó durante toda la vida de la revista, incluso tras el fallecimiento de Díez Gaviño, la base financiera para la publicación. Pero desde el principio quedó evidenciado que en la publicación se incorporaba un tercer pilar, la Asociación Vasco-Navarra de Beneficencia que funcionaba en la capital cubana desde 1878, y de la que se convirtió en portavoz semioficial de sus actividades, hasta el punto de incorporar en su mancheta de cabecera el escudo adoptado por la asociación (Álvarez Gila, 2019).

Otro buen ejemplo de esta práctica es la revista vasco-francesa Eskual Herria, estudiada por Mehats (2012: 448), quien señala que el primer testimonio sobre la existencia de esta revista, que le llevó a su redescubrimiento, fue una solicitud hecha por la junta directiva del Centre Basque Français de Buenos Aires a quien era su propietario, el periodista Laurent $\mathrm{H}$. Londaïts. Junto con los otros dos miembros de la plantilla (L. Araneder, redactor y Jean Darroget, administrador), todos ellos eran al mismo tiempo miembros de dicho centro vasco-francés. Como recoge Mehats, "il est important de souligner que le journal Eskual Herria et le centre basque français étaient très liés et que la rédaction du journal apprecia beaucoup le club et ses activités" $(2012,451)$, si bien es cierto que la dirección de la revista llevó una línea editorial independiente de la dirección del centro, llegando a producirse casos de distanciamiento entre ambos, como ocurrió con ocasión de la publicación de diversas noticias críticas que no agradaron a la directiva en 1900 y 1908, que llevaron incluso a la expulsión temporal de los responsables del Eskual Herria como asociados.

Paralelamente, también desde la prensa institucional de los centros vascos se desarrolló, por lo general, una evolución similar que tendía hacia una convergencia ambos tipos de publicaciones. Estas incursiones de los que inicialmente eran concebidos como meros boletines internos hacia un modelo de información más generalista dirigido a un público que iba más allá de los límites estrictos de la masa societaria, además, se pueden apreciar ya en momentos muy tempranos. Así, por ejemplo, ocurrió con la primera revista titulada Laurak Bat, la del centro vasco homónimo de Montevideo. Como señala Irigoyen Artetxe, si bien "esta revista" fue concebida para que sirviera como "nexo entre la sociedad y sus miembros", en sus páginas también: 
(..) se incluía la sección "Se desea saber el paradero de", utilizada generalmente por los recién llegados que traían mensajes para quienes, radicados anteriormente en la república, habían perdido contacto con sus lejanos parientes. Sirvió también para ubicar a personas perdidas en la inmensidad de los campos uruguayos y argentinos, ya que sus números era remitidos a la sociedad homónima de Buenos Aires. (Irigoyen Artetxe, 2000: 80).

También en Montevideo apareció el que podemos considerar el ejemplo más evidente de esta proyección externa de un órgano de prensa promovido y costeado económicamente por un centro vasco. Nos referimos a la revista Euskal Erria, creada en 1912 por el centro vasco homónimo. Un recorrido por los subtítulos que aclaraban al lector los objetivos y alcance de esta empresa periodística. A partir de su segundo número comenzó a ser referida como "Órgano Baskongado del Uruguay" ${ }^{10}$, pasando a ser nombrada como "Revista Baskongada del Uruguay" en $1914^{11}$. Este cambio de "órgano" a "revista", semánticamente muy significativo, se mantendría por varias décadas, con cambios que simplemente se referían a su periodicidad: "semanal" en $1914^{12}$, "decenal" en $1915^{13}$, "quincenal" en $1922^{14}$ y "mensual" en $1946^{15}$. No sería hasta 1952 que pasaría a recuperar de manera más evidente su vinculación al centro vasco, al convertirse en la "Revista oficial de la Sociedad Euskal Erria"16.

\section{La prensa política e ideológica}

Ya a comienzos del siglo XX se le uniría a los dos anteriores un tercer tipo de prensa de la diáspora vasco-americana: la prensa política. En gran medida, por no decir exclusivamente, se hallaba promovida por personas y asociaciones vinculadas al naciente nacionalismo vasco que, por aquellos mismos años, comenzaba a obtener un arraigo creciente en el panorama ideológico de la sociedad vasca. y que se estaba extendiendo hacia América (Núñez Seixas, 2017) Ya desde su nacimiento, el nacionalismo vasco vio en la prensa "el instrumento más eficaz de propaganda y por ello dedicó mucha energía y dinero a la creación de publicaciones" (Ruiz, 2008: 1). Así, el nacionalismo pronto entendería las colonias de emigrantes vasco-americanas como

10 № 2, de fecha 11 de agosto de 1912.

11 № 105, de fecha 2 de agosto de 1914.

12 № 107, de fecha 16 de agosto de 1914.

13 № 153, de fecha 10 de julio de 1915.

14 № 405, de fecha 15 de julio de 1922.

15 № 915, de fecha 1 de enero de 1946.

16 № 991, de fecha mayo-junio de 1952. 
un espacio privilegiado para el reclutamiento, la obtención de recursos financieros y la difusión ideológica (Álvarez Gila y Tápiz Fernández, 1996); de este modo, "la prensa se convirtió en imprescindible para difundir el nacionalismo entre la diáspora vasca" (Zelaia, 2018: 33). Protagonista de los primeros momentos de implantación de esta prensa política nacionalista fue la revista Irrintzi, de la mano del guipuzcoano Nemesio Olariaga, quien usó su fortuna personal, que había obtenido tras emigrar a la edad de quince años a Argentina:

El 1 de diciembre de 1903, aprovechando que su buen amigo Sebastián de Amorrortu había montado una imprenta (...) Nemesio Olariaga publicó con el título de Irrintzi y el subtítulo de "Dana Euskalerriagatik eta Euskalerriantzako"17 el primer periódico de carácter nacionalista en América. Posteriormente, en el año 1913, cambiaría este subtítulo por "Jan Goikoa eta Lege Zarrak"18 y "VIVA EUZKADI LIBRE".

Este periódico se editaba mensualmente (..). Constaba de ocho páginas y Nemesio Olariaga, aprovechando las rentas que le producían las fincas, se encargaba de los gastos y distribuía totalmente gratis y a su cuenta entre no solamente la colonia vasca allí existente, sin oque cientos y miles de ejemplares fueron saliendo por correo, muchos vía Filipinas, destinados a otros tantos vascos de Euskadi. "Esta publicación no acepta donativos ni suscripciones. Se envía de forma gratuta a todas las personas que lo soliciten". (Irusta Orbegozo, 2008: 81-82)

En agosto 1907, siguiendo la senda del Irrintzi, nacía en Rancagua (Chile) la revista mensual iAurrera!, que alternó en sus números -nos consta su existencia hasta 1910los mismos subtítulos que su correspondiente argentina. Al igual que aquella, también era la obra personal de un mecenas, identificado como Valentín de Landeta, director y redactor único, debido a que como se reconocía en su primer número en Chile, "habiendo muchos baskos, no existe, ni un círculo ni una sociedad para poder reunirse los hijos de Aitor"19 , y añadía bajo el epígrafe de "Nuestro Programa" que:

(..) en una palabra defenderemos la libertad amplia de nuestro Pueblo; y para ello haremos propaganda para la formación de un partido baskongado, predicando la concordia entre los baskos, para unirnos en un solo ideal "La Libertad de Euskadi" 20 .

17 "Todo por el País Vasco y para el País Vasco".

18 "Dios y Fueros".

19 "iAurrera mutillak!", iAurrera!, Rancagua, año 1, no 1, agosto de 1907, p. 1.

20 "Nuestro Programa", iAurrera!, Rancagua, año 1, no 1, agosto de 1907, p. 1. 
En todo caso, aunque tanto Irrintzi como iAurrera! parecen ser la obra de francotiradores, son también reflejo de un creciente proceso de recepción del nacionalismo en las colonias vascas de América. Salazar González y Hernández Ponce, por ejemplo, definen la creación de iAurrera! como una consecuencia directa de la introducción del nacionalismo en la colonia vasca de Chile, debida a la labor de sacerdotes y religiosos vascos (menciona capuchinos, cordimarianos, escolapios, entre otros) que fueron "inequívocos exponentes de una identidad nacional vasca que unía el catolicismo y sus compromisos morales a la condición de vasco" y que por este motivo "proponían y enseñaban conceptos de nacionalismo sabiniano radical" (Salazar González y Hernández Ponce, 2007: 211-212).

Si bien la prensa vasco-americana preexistente, por lo general, no se mostró inicialmente contraria a aceptar en sus páginas notas y artículos firmados por autores próximos al nacionalismo - de hecho, fue esto mismo una de las causas principales de la ruptura entre los cofundadores de La Vasconia, al negarse Uriarte a censurar tales artículos frente a las presiones de Grandmontagne-, con la creación de las primeras instituciones asociativas del nacionalismo político en el seno de la colonia vasca se reprodujo pronto la misma práctica de que estas nuevas entidades promovieran también la creación de sus propios boletines y revistas. En 1911 nacía en la ciudad de Rosario, Argentina, el centro vasco Zazpirak Bat, el primero que por nombre, fines y promotores era ya plenamente nacionalista. Detrás de su fundación se hallaba un grupo denominado "Comité Nacionalista Vasco", que funcionaba oficiosamente como una delegación extraterritorial del Partido Nacionalista Vasco (Álvarez Gila, 2000). Hasta mediada la década de 1920 este centro vasco ensayó la publicación de sucesivas revistas, de corta duración, todas ellas inspiradas -incluso en su nombre-en el ideario nacionalista: Aitor (1914), Egizale (1915), Patria (1917) y Denak Bat (1922). Paralelamente en Buenos Aires se creaba otra delegación, esta vez formalmente constituida, del mismo partido nacionalista, que acabaría siendo denominada Acción Vasca de Argentina debido a impedimentos legales. Alrededor de este núcleo surgirían otras empresas periodísticas: Euzkotarra (1913), Euzko Alderdi Abertzalia (1920) y, finalmente, Nación Vasca (1924), que saldría a la calle con regularidad hasta 1937. También las colonias vascas en otros países, como México o Estados Unidos, vieron el nacimiento de iniciativas muy similares en las décadas de 1920 y 1930.

Esta prensa nacionalista vasca de América, además, se hallaba en estrecho contacto con su contraparte en el propio País Vasco. Esto no solamente se aprecia en la transferencia de modelos estéticos: por ejemplo, el primer Euzkotarra de Buenos Aires presentaba una maquetación muy similar al diario Euzkadi de Bilbao, que fue hasta la Guerra Civil el principal diario nacionalista vasco. También se percibe en la transferencia de artículos: mientras la prensa nacionalista en América solía reproducir artículos previamente aparecidos en periódicos en Europa, muchos de sus redactores se convirtieron en auténticos corresponsales oficiosos de periódicos como Euzkadi o 
El Día (la principal cabecera nacionalista en San Sebastián). "Lautzi" desde Chile, la redacción de Nación Vasca desde Argentina, "Etxeberria'tar" desde México o el director del programa radiofónico -y posterior publicación- Euskal Ordua desde Uruguay, nutrían las páginas de la prensa nacionalista en el País Vasco de informaciones sobre la actualidad del movimiento nacionalista vasco en America (Álvarez Gila, 2005: 380. Irigoyen Artetxe, 2010b).

\section{La prensa vasco-americana y la definición de la identidad diaspórica vasca}

Un último elemento sobre el que quisiéramos esbozar un análisis preliminar se relaciona con el modo en el que la prensa vasco-americana influyó a la hora de determinar la definición de la identidad diaspórica vasca. Entendemos por identidad diaspórica vasca el conjunto de rasgos que las comunidades formadas por los vascos emigrados y sus descendientes fueron cristalizando, a lo largo de sucesivas décadas, como sus partes constituyentes, derivada pero no similar a la que simultáneamente se estaba conformando en el propio País Vasco. En América la identidad vasca se ha venido a constituir, en su fase actual, en un conglomerado que une por un lado la conjunción de rasgos originales de la cultura vasca con las particulares experiencias de los colectivos inmigrantes en su país de destino, por otro lado la pervivencia de rasgos arcaizantes que han sido transformados y simplemente han desaparecido en la patria de origen, y finalmente, una definición postnacionalista de los límites de dicha identidad que integra en su imaginario a todos los territorios tradicionalmente identificados con la cultura vasca, a ambos lados de la frontera vasco-española (Álvarez Gila, 2016).

En este contexto, la prensa debió jugar un papel central, siendo al mismo tiempo un elemento catalizador de esta evolución por ser un ámbito de transmisión y debate de discursos, incluidos los identitarios, y un testimonio histórico de tal transmisión y debate. $\mathrm{Y}$, en todo caso, constituye una ventana que nos permite vislumbrar elementos para la comprensión del proceso de creación y evolución identitaria de las comunidades vasco-americanas. A este respecto, la misma aparición de un sistema de publicaciones periódicas propias y privativas para los colectivos vascos ya constituía, en sí, un primer e importante paso en la visibilización exterior y cohesión interna de dichos colectivos. Moya, por ejemplo, analizando el caso de los españoles pone en relación la diversificación regional que en Argentina -al igual que en otros países receptores de inmigración- experimentan tanto las instituciones asociativas que 
agrupan a los inmigrantes como el proceso paralelo que ocurre en el periodismo: "EI periodismo no demostró ser inmune a lo que un sociólogo español visitante denominó 'el fraccionamiento insolidario'. Treinta y dos de las setenta publicaciones de inmigrantes que se catalogan (..) representan a regiones ibéricas, y seis de ellas se publicaban en lenguas locales, no en castellano" (Moya, 2004: 305).

No obstante, cuando dichas instituciones e iniciativas periodísticas surgen, para el caso de los vascos, en la década de 1870 en lugares tan distantes como Buenos Aires, Montevideo y La Habana, un análisis cruzado de las referencias identitarias y de los marcos nacionales de referencia que presentaban de forma explícita e implícita estaban lejos de mostrar una ruptura con las identidades políticas española o francesa, según su caso, con una comprensión de lo vasco como un elemento marcadamente regionalista dentro del conjunto de España. Así lo plasmaba, por ejemplo, la mancheta de cabecera de la revista Laurak Bat de Buenos Aires en sus primeros números -en los que aún aparece con una grafía más próxima a la norma castellana, usando la "c" en vez de la "k"-. En su número 1 se presenta con el subtítulo de "Revista de la Sociedad vasco-navarra de Buenos Aires", colocando como elemento icónico una representación de un escudo imperial español (Álvarez Gila, 2019).

Algo similar se aprecia pocas décadas más tarde, cuando nacen los primeros órganos de prensa para el grupo vasco-francés. Mehats, realizando un análisis del contenido del Eskual Herria de Buenos Aires (década de 1890) llega a una conclusión similar. No es por casualidad que se presente en el sutbítulo como "Journal Basque Français du Rio de la Plata": Mehats $(2012,454-457)$ aprecia que hay un predominio de referencias (en noticias, descripciones, artículos históricos, culturales, etc..) sobre el País Vasco-Francés, aunque esto no obsta para que también se incluyan menciones sobre vasco-españoles, si bien limitadas a cuestiones de cultura y literatura en lengua vasca. De hecho, como lema del periódico sus promotores adoptaron los conocidos versos del guipuzcoano José María de Iparragirre: "Herri alde guzietan yende onak badira, bainan bihotzak dio zoaz Eskual Herrira". Durante la guerra hispano-cubana de 1898, por ejemplo, mientras que la prensa vasco-española de Argentina se posicionaba a favor de los derechos de la metrópoli, en el Eskual Herria por el contrario abundaban los artículos que defendían el derecho "de un pueblo que lucha por su libertad". Así "En langue basque, Laur Begui avec le titre 'Bakhea' (La paix) explique le conflict cubain et les positions diplomatiques des grandes puissances. Mendi Hurbil en langue basque s'inquiète aussi que la guerre d'indépendance des Cubains contre l'Espagne soit compliquée par l'appétit des États-Unis d'Amérique" (Mehats, 2012: 457). En todo caso, cuando los artículos derivaban hacia cuestiones vinculadas a la política francesa, su visión era claramente regionalista. En su artículo "Basques Français, se afirma que la independencia es una utopía y la autonomía una 
quimera, y pone el futuro de la lengua vasca en la transmisión maternal, porque admite como beneficiosa la aculturación nacional francesa ${ }^{21}$.

Hubo, sin embargo, ya desde la década de 1880 algunas voces que, sin negar el regionalismo propio de la identidad vasca (ya fuera española o francesa), planteaban al mismo tiempo una definición identitaria que hoy denominaríamos como de "nacionalismo cultural", que propugnaba el mantenimiento y la intensificación de los lazos entre ambos grupos vascos, nor- y sud-pirenáicos, en un contexto de coexistencia en un mismo espacio, los países americanos. El cambio comenzó en la comunidad vasca de Uruguay con el nacimiento de una nueva euskal etxea, el Centro Vascongado de Montevideo, en 1885. En el único ejemplar que se ha conservado de su boletín homónimo, hacían una presentación pública de sus objetivos, cuyo primer punto pasaba por "estrechar los vínculos de unión y fraternidad entre los vascongados de ambas vertientes de los Pirineos y sus descendientes que residan en el país"22. Posteriormente, en las décadas de 1890 a 1910, otros centros vascos de Argentina, Uruguay, Chile y Cuba seguirían la misma senda de propugnar la unidad de todos los vascos, sin un significado político como el que adquiriría, a partir de ese momento, con la introducción del nacionalismo vasco en su formulación ideológica clásica definida por Sabino Arana.

Sería preciso estudiar, a este respecto, los elementos que dieron pie y sirvieron como justificación a este proceso. Entre ellos destacaba, sin duda, la lengua vasca como rasgo visible común entre ambos grupos. Curiosamente, fue entre los vascos franceses donde se dio una mayor tendencia a introducir el idioma vasco como elemento presente de forma habitual y destacada en sus páginas. Los vascos del norte de la frontera franco-española tenían una tradición más consolidada del uso literario del euskara, debido sobre todo al papel de la Iglesia católica, lo que se traducía en una mayor facilidad para las habilidades lectoescritoras en esta lengua. El primer número del Eskual Herria de Buenos Aires, por ejemplo, publicó en euskara dos de las tres notas de su portada, reservando además para este idioma la más, importante, es decir la presentación de los objetivos la revista ("Gure Chedea"). Entre los vasco-españoles, por el contrario, se aprecia un menor uso práctico del idioma, si bien el euskara mantuvo siempre una función representativa: una especie de marchamo identificativo de grupo, función que se acrecentaría a lo largo del siglo XX por el uso de esta estrategia por parte de la prensa del nacionalismo vasco. Solo desde aquí puede comprenderse el error que comete Moya (2004: 336) cuando hablando de la introducción de los nacionalismos vasco, catalán y gallego en Buenos Aires, afirma que "los vascos adoptaron esta línea con mayor intensidad que cualquier otro grupo

21 "Basques Français", Eskual Herria. Journal Basque Français du Rio de la Plata, oo 5 (30 enero 1895), p. 1.

22 "El Centro Vascongado. Programa", El Centro Vascongado, no 1 (febrero 1885), p. 1. 
de inmigrantes ibéricos. Algunos publicaron periódicos separatistas en euskara, su lengua materna". Realmente el periódico al que se refiere, Aberu-Eguna (sic), supuestamente publicado entre 1932 y1933 en Buenos Aires ${ }^{23}$, efectivamente presentaba el título en lengua vasca, si bien lo cierto es que en ninguna de las publicaciones nacionalistas que se recogen en América desde 1907 en adelante conocemos un caso en el que la lengua vasca fuera la única, o incluso la mayoritaria, en sus páginas.

En todo caso, para fines de la década de 1920 y a lo largo de la década siguiente, el panorama de la prensa diaspórica vasca había alcanzado un estadio de madurez, en el que junto a una variedad de cabeceras y lugares de publicación, las diversas publicaciones mostraban una gran diversidad. Coexistían de este modo revistas privadas junto con boletines institucionales de los centros vascos, existían unas corrientes de contacto y transmisión, no solo con la prensa en el País Vasco, sino entre las diversas publicaciones vasco-americanas, y finalmente, con la llegada de la II República en España y el renovado interés por el debate político surgieron, incluso, instituciones y revistas que refutaban el proceso de unificación entre vascos españoles y franceses y seguían reivindicando el regionalismo como pilar de la identidad vasca, tal y como fue el caso del Centro Euskaro Español -y su correspondiente revista mensual- de Montevideo, que en su primer número de abril de 1924 reafirmaba su objetivo de "difundir, valiéndose de los medios que considere más eficaces, el conocimiento de las provincias [de Alava, Vizcaya, Guipúzcoa y Navarra], (..) así como ensalzar el nombre de España, rindiendo el honor debido a sus glorias e hijos más preclaros" 24 . El estallido de la guerra y la posterior llegada del exilio republicano y nacionalista modificaría, en las siguientes décadas, este panorama.

\section{Bibliografia}

ALONSO, Paula, comp. (2004). Construcciones impresas. Panfletos, diarios y revistas en la formación de los estados nacionales en América Latina, 1820-1920. Buenos Aires, Fondo de Cultura Económica.

ÁlVAREZ, Adriana, Mauro VITULLO y Laura ECHEZARRETA (2009). "Los vascos en la prensa americana: recopilación y digitalización de noticias sobre el País Vasco y

23 Decimos "supuestamente" porque en la hemerotca no consta ninguna publicación periódica con dicho título en las fechas indicadas. Creemos, en todo caso, que no se refiera aquí sino a la serie de ediciones conmemorativas al hilo de la celebración anual del Día de la Patria Vasca, que es lo que significa "Aberri Eguna" (y no "Aberu-Eguna", que es una evident incorrección).

24 "Objeto de la Sociedad", Centro Euskaro Español, año 1, no 1 (abril de 1924), pp. 1-2. 
las colectividades vascas", Nexos. Universidad Nacional de Mar del Plata, 16:26, pp. 39-44.

ÁLVAREZ GILA, Óscar (2000). "Los inicios del nacionalismo vasco en Rosario (Argentina)", Sancho el Sabio, 12, pp. 153-176.

- (2005). "Las nuevas Euskal Herrias americanas: los vascos y las emigraciones ultramarinas (1825-1950)". En AGIRREAZKUENAGA ZIGORRAGA, Joseba (dir.); La crisis de la civilización de los vascos del Antiguo Régimen y estrategias de revolución liberal e industrial: 1789-1876, Donostia-San Sebastián, Editorial Lur, pp. 319-391.

- (2016). "De una emigración regional a una colectividad nacional. Imagen y procesos en la construcción identitaria de la diáspora vasca en el Río de la Plata", RiMe. Rivista dell'Istituto di Storia dell'Europa Mediterranea, 17/1, pp. 63-85.

- (2019). Antes de la ikurriña. Banderas, símbolos e identidad vasca en América (1880-1935). Madrid, Ediciones Sílex.

ÁLVAREZ GILA, Óscar y José María TÁPIZ FERNÁNDEZ (1996). "Prensa nacionalista vasca y emigración a América (1900-1936)", Anuario de Estudios Americanos, LIII:1, pp. 233-260.

ARBELBIDE, Xipri (2001). "Californiako Eskual Herria". En LARRONDE, Jean-Claude (ed.). Eugène Goyheneche Omenaldia - Hommage. Donostia-San Sebastián, Eusko Ikaskuntza, pp. 17-51.

- (2003). Bidegileak: Jean Pierre Goytino (1860-1920). Vitoria-Gasteiz, Eusko Jaurlaritzaren Argitarapen Zerbitzu Nagusia.

- (2006). "Jean Pierre Goytino, Los Angelesetako notarioa", Jado. Boletín de la Academia Vasca de Derecho, 9, pp. 117-125.

ARES, Pedro (2004). Grandmontagne. El escritor vascoespañol que se inició en la Pampa a fines del siglo XIX. Buenos Aires, Editorial Mainz.

AZCONA PASTOR, José Manuel (1992). Los paraísos posibles. Historia de la emigración vasca a Argentina y Uruguay en el siglo XIX. Bilbao, Universidad de Deusto.

BERGARECHE, Macarena (2009). "El ser vasco en un país lejano. Identidad e integración. La Vasconia (1893-1914)", Temas de historia argentina y americana, 15, pp. 15-51.

CAVA MESA, Begoña (1996). "El asociacionismo vasco en Argentina. Política cultural", en ESCOBEDO MANSILLA, Ronald et alii, eds. Emigración y redes sociales de los vascos en América, Bilbao, Universidad del País Vasco, pp. 137-169. 
DE DIOS ALTUNA DE MARTINA, Ángeles (2011). Andrea Moch. Andanzas de una artista. Buenos Aires, Dunken.

- (2012). "Indalecio Ojanguren fotógrafo: su obra en la revista éuskaroargentina La Baskonia (1913-1935)", Vasconia, 38, pp. 645-688.

- (2012b). "José Rufo de Uriarte, periodista, editor y escritor", Euskonews, no 642, 643 y 644. Disponible en Internet (4-3-2019): http://www.euskonews.eus/0642zbk/kosmo64201es.html

- y Óscar ÁLVAREZ GILA (2010). "José R. de Uriarte y la revista La Baskonia: una visión atípica de la colectividad vasca de Argentina de entre siglos". En GARCíA SEBASTIANI. Marcela, dir. Patriotas entre naciones. Élites inmigrantes españolas en Argentina (1870-1940). Madrid, Editorial Complutense, pp. 339-359.

DÍAZ-NOCI, Javier (1994). Euskarazko aldizkari, egutegi eta almanaken erroldea (1834-1959). Donostia-San Sebastián, Eusko Ikaskuntza.

- (1995). Euskal prentsaren sorrera eta garapena (1834-1939), Donostia-San Sebastián, Eusko lkaskuntza.

- (2001). "Historia del periodismo en lengua vasca de los Estados Unidos. Dos semanarios de Los Ángeles en el siglo XIX", Zer. Revista de Estudios de Comunicación, 10, disponible en Internet (4-3-2019): http://www.ehu.eus/ojs/index.php/Zer/article/view/6120/5802

EZKERRO, Mikel (2003). Historia del Laurak Bat de Buenos Aires. Vitoria-Gasteiz, Gobierno Vasco.

IRIGOYEN ARTETXE, Alberto (2000). "Laurak Bat de Montevideo. Primera euskal etxea del mundo", Gerónimo de Ustáriz, 16, pp. 73-91.

- (2010). "Publicaciones periódicas vascas en Uruguay (1876-1939)". En ÁLVAREZ GILA, Óscar (dir.). Organización, identidad e imagen de las colectividades vascas de la emigración (siglos XVI-XXI). Bilbao, Universidad del País Vasco, pp. 207-221.

- (2010b). "La difusión radiofónica del ideario nacionalista vasco en Uruguay: la Comisión Pro Fomento Euskal Ordua de Montevideo. 1932-1936", Mediateka, 12, pp. 257-299.

IRUSTA ORBEGOZO, Juan María (2008). "Biografía de Nemesio Olariaga", Leiçaur, 10, pp. 1-46.

MÁRQUEZ ORTIZ, Reyes (1996). "Colectividad vasca y asociacionismo en Argentina", en ESCOBEDO MANSILLA, Ronald et alii, eds. Emigración y redes sociales de los vascos en América, Bilbao, Universidad del País Vasco, pp. 123-136. 
MEHATS, Claude (2002). "Articles en basque parus en 1843 dans le Patriote Français de Montevideo", Lapurdum, 7, pp. 247-257.

- (2005). Organisation et aspects de l'émigration des Basques de France en Amérique, 1832-1976. Vitoria-Gasteiz, Gobierno Vasco.

- (2012). "Eskual Herria: journal des basques français du Río de la Plata", Vasconia, 38, pp. 447-478.

MOYA, José C. (2004). Primos y extranjeros. La inmigración española en Buenos Aires, 1850-1930. Buenos Aires, Emecé Argentina.

NÚÑEZ SEIXAS, Xosé Manoel (2017). "¿Negar o reescribir la hispanidad? Los nacionalismos subestataales ibéricos y América Latina, 1898-1936", Historia Mexicana, LXVII:1, pp. 401-458.

PEREA BEOBIDE, Aroa (2017). "On the Beginnings of Basque-American Press: An Analysis on the Newspaper Californiako Eskual Herria (1893)". Trabajo de Fin de Grado (Historia). Facultad de Letras, Universidd del País Vasco. Disponible en Internet (4-3-2019): https://addi.ehu.es

RAMOS MARTÍNEZ, Jon Ander (2010). "Los inicios de la prensa vasca en Cuba. Laurac Bat de La Habana (1886-1895)". En ÁLVAREZ GILA, Óscar (dir.). Organización, identidad e imagen de las colectividades vascas de la emigración (siglos XVI-XXI). Bilbao, Universidad del País Vasco, pp. 195-206.

- (2011). "Faustino Díez Gaviño e a primeira empresa xornalística en Cuba", Estudos Migratorios: revista galega de análise das migracións, 4:1-2, pp. 85-104. - (2017). "Una aproximación a la prensa periódica en la diáspora vascoamericana (siglos XIX-XX)". En GARABEDIAN, Marcelo Hugo, comp. La prensa periódica española en América Latina: voces, prácticas y diálogos para la identidad española en la diáspora, Buenos Aires, Leviatán, pp. 80-100.

RUIZ, Nicolás (2008). "La prensa nacionalista en Vizcaya durante la Restauración: el espejo de una comunidad en construcción". El Argonauta Español, 5, disponible en Internet (4-3-2019): http://argonauta.revues.org/970

SALAZAR GONZÁLEZ, Julene y Roberto HERNÁNDEZ PONCE (2007). "Del Centro Vasco al Euzko Etxea de Santiago de Chile". En OYANGUREN, Pedro, ed.lit. Vascos en Chile, 1520-2005. Euzko Etxea de Santiago, Donostia-San Sebastián, Eusko Ikaskuntza, pp. 199-255.

TORREGROSA CARMONA, Juan Francisco (2015). "Un siglo de prensa vasca en América". En AZCONA PASTOR, José Manuel (ed.lit.). Identidad y estructura de la emigración vsca y navarra hacia Iberoamérica, siglos XVI-XXI. Redes sociales y desarrollo socioeconómico, Madrid, Thomson Reuters Aranzadi, pp. 362-382. 
TOTORICAGUENA, Gloria P. (2005). Comparing the Basque Diaspora. Ethnonationalism, Transnationalism and Identity Maintenance in Argentina, Australia, Belgium, Peru, the United States of America, and Uruguay. Tesis doctoral. The London School of Economics and Political Sciences. Unieversity of London, disponible en https://search.proquest.com/docview/301558031

ZELAIA, Kepa (2018). Ameriketako diasporako euskal prentsari buruzko azterketa: 1877-1977. Trabajo de Fin de Máster. Máster en Historia Contemporánea, Universidad del País Vasco. Disponible en Internet (4-3-2019): http://addi.ehu.eus

\section{Fuentes}

IRIGOYEN ARTETXE, Alberto y Adriana PATRÓN (2003). Documentos. Revista EuskaroAmericana La Baskonia (1893-1943). Vitoria-Gasteiz, Gobierno Vasco (=Colección Urazandi, no 0).

IRIGOYEN ARTETXE, Alberto y Adriana PATRÓN (2007). Hemeroteca de la Diáspora Vasca. Vitoria-Gasteiz, Gobierno Vasco, 10 vols. Disponible en Internet (4-32019): http://urazandi.euskaletxeak.net 